Full toroidal imaging of non-axisymmetric plasma material interaction in the National Spherical Torus eXperiment divertor

F. Scotti, A. L. Roquemore, V. A. Soukhanovskii

May 8, 2012

Review of Scientific Instruments 
This document was prepared as an account of work sponsored by an agency of the United States government. Neither the United States government nor Lawrence Livermore National Security, LLC, nor any of their employees makes any warranty, expressed or implied, or assumes any legal liability or responsibility for the accuracy, completeness, or usefulness of any information, apparatus, product, or process disclosed, or represents that its use would not infringe privately owned rights. Reference herein to any specific commercial product, process, or service by trade name, trademark, manufacturer, or otherwise does not necessarily constitute or imply its endorsement, recommendation, or favoring by the United States government or Lawrence Livermore National Security, LLC. The views and opinions of authors expressed herein do not necessarily state or reflect those of the United States government or Lawrence Livermore National Security, LLC, and shall not be used for advertising or product endorsement purposes. 


\title{
Full toroidal imaging of non-axisymmetric plasma material interaction in the National Spherical Torus eXperiment divertor ${ }^{\mathrm{a}}$
}

\author{
Filippo Scotti, ${ }^{1, b)}$ A.L. Roquemore, ${ }^{1}$ and V. A. Soukhanovskii ${ }^{2}$ \\ 1) Princeton Plasma Physics Laboratory, Princeton, NJ, USA \\ ${ }^{2)}$ Lawrence Livermore National Laboratory, Livermore, CA, USA
}

(Dated: 5 May 2012)

A pair of two dimensional fast cameras with a wide angle view (allowing a full radial and toroidal coverage of the lower divertor) were installed in the National Spherical Torus eXperiment (NSTX) in order to monitor nonaxisymmetric effects. A custom polar remapping procedure and an absolute photometric calibration enabled easier visualization and quantitative analysis of non-axisymmetric plasma material interaction (e.g. strike point splitting due to application of 3D fields and effects of toroidally asymmetric plasma facing components).

\section{INTRODUCTION}

In magnetic confinement devices, a divertor configuration is employed to concentrate the plasma material interaction away from the core plasma interface. In diverted tokamaks, toroidal axisymmetry for particle and heat fluxes at the divertor plates is usually assumed. However, there are often cases when this assumption is not valid.

In NSTX, lithium coatings are applied on the lower divertor plasma facing components (PFCs) as a wall conditioning technique by means of two lithium evaporators, called LITERs and separated toroidally by $150^{\circ 1}$. Four toroidal segments of a porous molybdenum surface (each covering about $80^{\circ}$ toroidally and separated by an ATJ graphite tile) were installed in the outer divertor region ${ }^{2}$. These molybdenum PFCs with heating capabilities (Liquid Lithium Divertor, LLD) were designed in order to maintain a liquid lithium surface at the plasma-wall interface. The capability of independently heating the LLD segments and the presence of different or unevenly coated PFCs are a clear example of possible non-axisymmetric plasma material interaction. Furthermore, in NSTX the application of $3 \mathrm{D}$ magnetic perturbations $(n=1,3)$ was shown to result in strike point splitting, with the outer strike point (OSP) separating in helical lobes of heat and particle fluxes ${ }^{3}$.

In order to study non axisymmetric effects in the NSTX divertor, two fast two dimensional cameras with a wide angle view were installed, giving a full radial and toroidal coverage of the lower divertor. In this paper, the setup, calibration and applications are presented.

\section{EXPERIMENTAL SETUP}

In order to enable a wide angle view of the lower divertor, two re-entrant view ports (3" outer diameter, $1 / 8$ " thick sapphire window at the end of a 3.5 " outer diameter,

\footnotetext{
a) Contributed paper published as part of the Proceedings of the 19th Topical Conference on High-Temperature Plasma Diagnostics, Monterey, California, May, 2012.

b)fscotti@pppl.gov
}

5" long pipe) were developed, located $150^{\circ}$ apart on the top of the vacuum vessel on Bay E and Bay J (in NSTX each bay is separated by $30^{\circ}$ toroidally, A-L). Views of the NSTX lower divertor as imaged by the two cameras are shown in Figure 1: a) the view from Bay E and c) the view from Bay J. The typical resolution of $256 \times 208$ pixels gives a spatial resolution of $\sim 0.8 \mathrm{~cm}$. While two different cameras are used for the two view ports, the same setup (shown in Figure 2) is adopted. In each setup, light is collected by a $\mathrm{f}=16 \mathrm{~mm} \mathrm{C}$-mount lens (Pentax, F/1.4) which provides the desired wide angle view $\left(\sim 44^{\circ}\right)$, with a clearance of $1 / 8$ " between the optics and the re-entrant window. The collected light is imaged on a coherent optical fiber bundle (Schott IG-163) 15 feet in length, which consists of $1000 \times 80010 \mu \mathrm{m}$ fibers. A f $=50 \mathrm{~mm}$ C-mount lens (Pentax, F/1.4), installed at the camera end of the light guide, collimates the light rays while a $\mathrm{f}=25 \mathrm{~mm} \mathrm{C}$-mount lens (Pentax, F/1.4) focuses the image on the camera detector and provides the demagnification needed for the image to fill about $1 / 16$ of the sensor. Between the collimating and the focussing lens (both focussed at infinity) a filter wheel is used to change the narrow three cavity bandpass interference filter (ANDOVER ${ }^{4}, \Delta \lambda \sim 1.5 \mathrm{~nm}$ ) through which the plasma is imaged. A list of the filters used including spectral lines and transitions, is shown in Table 1. Both filter wheels are remotely controlled by a Visual Basic application that allows for the interchange of filters between discharges. As mentioned above, two different cameras are employed. The Phantom v710 has a $1280 \times 800$ pixel sensor and a $25.6 \times 16.0 \mathrm{~mm}$ sensor area with a 12 bit dynamic range and a maximum frame rate (at the resolution of $256 \times 208$ ) of 100,000 frames per second (fps). The Phantom v7.3 has a $800 \times 600$ pixels sensor and a $17.6 \mathrm{~mm} \times 13.2 \mathrm{~mm}$ sensor area with a 14 bit dynamic range and a maximum frame rate of 60,000 fps (at the resolution of $224 \times 184$ ). Each camera is controlled by a Labview VI via a private 1 Gigabit fiber optic link that allows the full transfer of the data within the shot cycle ( 1-10GB/discharge/camera).

The wide angle view of the imaging lenses, the use of re-entrant ports and the small major radius of NSTX allowed the imaging of over $50 \%$ of the toroidal extent of the divertor on each camera. Remapping the camera data in polar coordinates (radius vs toroidal angle) can be a convenient tool for a better visualization and an easier comparison of radial profiles at different toroidal locations. The intersection of the conic surfaces defined 
by the collection optics with the divertor plane results in elliptical conic sections. Conic surfaces with increasing subtended angle (intersecting the divertor at increasing radii) define ellipses with decreasing elongation. Barrel distortion can lead to a further change in the elongation for structures at different radii. A custom polar remapping routine was developed in order to correct for these effects. In a given field of view, up to five concentric
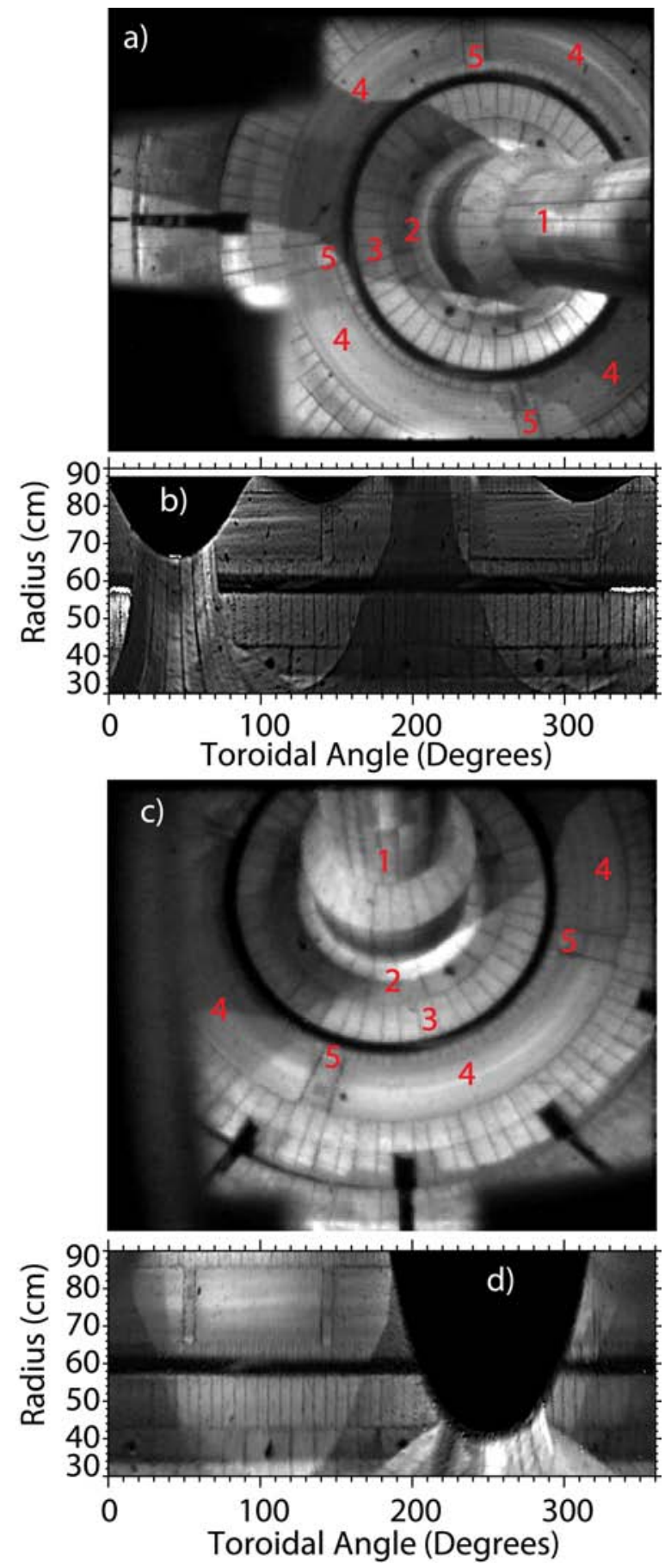

FIG. 1. Lower divertor view from Bay E (a) and Bay J (c); polar remapping of divertor view from Bay E (b) and Bay J (d). In the field of view one can observe: 1) center stack, 2) first row of graphite tiles, 3) second row of graphite tiles, 4) LLD, 5) diagnostic graphite tiles.

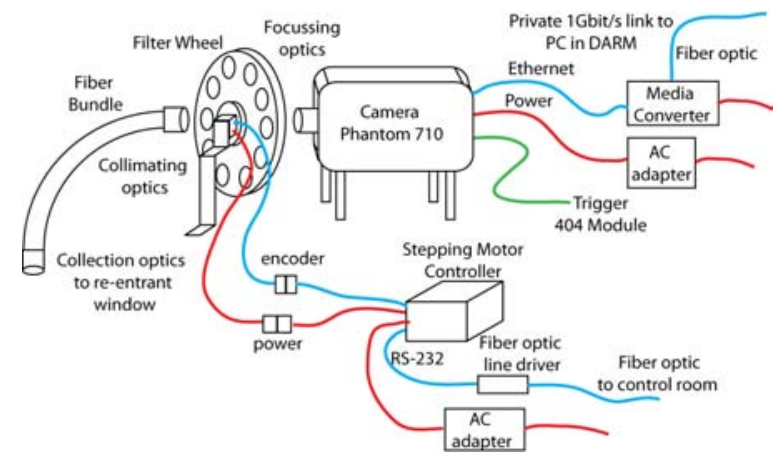

FIG. 2. Schematic of camera setup.

TABLE I. Bandpass filters and atomic transitions

\begin{tabular}{cccc}
\hline \hline$\lambda(\mathrm{nm})$ & $\Delta \lambda(\mathrm{nm})$ & Ionic species & Transition \\
\hline 656.5 & 1.0 & D (Balmer $\alpha)$ & $n=2-3$ \\
658.5 & 1.5 & C II & $1 s^{2} 2 s^{2} 3 s^{1}-1 s^{2} 2 s^{2} 3 p^{1}$ \\
465.0 & 1.5 & C III & $1 s^{2} 2 s^{1} 3 s^{1}-1 s^{2} 2 s^{1} 3 p^{1}$ \\
580.9 & 1.5 & C IV & $1 s^{2} 3 s^{1}-1 s^{2} 3 p^{1}$ \\
670.9 & 1.5 & Li I & $1 s^{2} 2 s^{1}-1 s^{2} 2 p^{1}$ \\
548.5 & 1.5 & Li II & $1 s^{1} 2 s^{1}-1 s^{1} 2 p^{1}$ \\
\hline
\end{tabular}

circular divertor structures (bottom of center stack, first and second graphite tile rows, inner and outer edges of LLD) are fitted to concentric ellipses with different elongations. A polar map is then created where, for every radius, a linear combination of the two closest reference ellipses is used. The camera data are remapped on the polar map and the toroidal angle is linearized to correct for lens distortion. Images of the lower divertor were routinely taken exploiting vessel illumination during the experimental campaign and used to create the polar map as shown in Figure 1 b) and d). Combining the two views, full toroidal imaging of the lower divertor is possible.

In order to quantitatively analyze $2 \mathrm{D}$ data, an absolute calibration is performed for the two cameras over the entire field of view. The calibration is carried out by means of a high reflectance lambertian target with uniform illumination (whiteplate) and an integrating sphere. Due to the small aperture of the integrating sphere (1") compared to the field of view, the relative sensitivity of the system is calibrated using the whiteplate. The whiteplate is positioned in-situ in three different locations and the three calibrations are combined to obtain the $2 \mathrm{D}$ relative sensitivity over the field of view. This is then absolutely scaled using the luminance of the integrating sphere for each filter. Inverting the acquired data by the sensitivity function, 2D absolutely calibrated data are obtained. The absolute brightness of each spectral line measured during experiments (photons $/ \mathrm{m}^{2} / \mathrm{s}$ ) can then be directly related to influxes of specific ionic species (ionizations $/ \mathrm{m}^{2} / \mathrm{s}$ ) using the $\mathrm{S} / \mathrm{XB}$ method $^{5}$. Here, $\mathrm{S} / \mathrm{XB}$ coefficients based on the ADAS database are used. 


\section{APPLICATIONS}

\section{A. Toroidal asymmetry in plasma facing components}

Full toroidal imaging enabled the analysis of how recycling and impurity influxes vary on different or differently coated materials as well as on PFCs at different temperatures.

Simulations show that the lithium evaporation geometry in NSTX can lead to a toroidal variation in the coating thickness up to a factor of $3^{6}$. Fresh coating thicknesses (15-45nm for a $200 \mathrm{mg}$ evaporation) are within the stopping range of typical divertor incoming ions. However, as a result of divertor ion fluxes, the characteristics of the coatings are expected to change during a discharge due to melting, evaporation, erosion, re-deposition and chemical reactions. Toroidal asymmetries in local lithium influxes are generally observed and are enhanced during transient divertor heat loads, such as ELMs. The transient heat load causes differential heating of coatings with different thickness and a consequently different lithium sputtering response ${ }^{7}$. In Figure 3 (top), the simulated lithium toroidal distribution is shown. Areas with heavier lithium deposition, as well as areas shadowed by the center stack can be easily seen. In Figure 3 (bottom), a full polar plot of $\mathrm{Li}$ I brightness in the divertor region is shown, obtained combining views from both Bay E and Bay J after a Type I ELM. Li I brightness is related to neutral lithium influx from the PFCs, via the S/XB coefficients. The two regions in which the LITERs are shadowed by the center stack result in a lower lithium influx and closely match the simulated distribution.

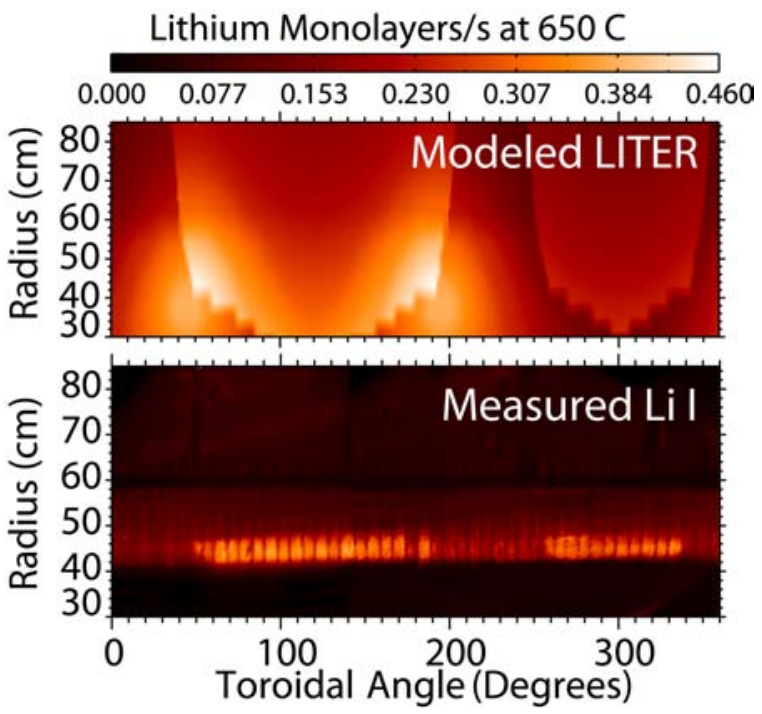

FIG. 3. Simulated fresh lithium coatings thickness (top) and measured Li I brightness from the lower divertor after an ELM event (bottom).

The response of differently heated PFCs was by externally heating three LLD segments at $220^{\circ} \mathrm{C}$ (above the lithium melting temperature, $180^{\circ} \mathrm{C}$ ) while the last one remained below $180^{\circ} \mathrm{C}$. In these discharges, the OSP was controlled at $\mathrm{R}=63 \mathrm{~cm}$, just inboard of the LLD. In Fig- ure 4 , the divertor region is imaged through $\mathrm{D}-\alpha$ (top), $\mathrm{Li}$ I (middle) and Li II (bottom) filters. Higher D $\alpha$ emission can be seen on the warm LLD plates (1,2,4 in Figure 4), while the cold LLD segment (3 in Figure 4) exhibits emission comparable to the nearby graphite tiles. Assuming toroidal symmetry in incident ion fluxes, this indicates increased recycling on the warm LLD plates. Heated LLD segments also clearly show enhanced neutral and singly ionized lithium influxes (up to $2 \times$ ). This can be interpreted as a result of the temperature dependence of lithium physical sputtering yield ${ }^{7}$. Again, the cold LLD segment shows influxes comparable to the nearby graphite tile. Furthermore, one can notice at the OSP location on the bullnose tile, the asymmetry in Li I emission due to uneven lithium coatings.

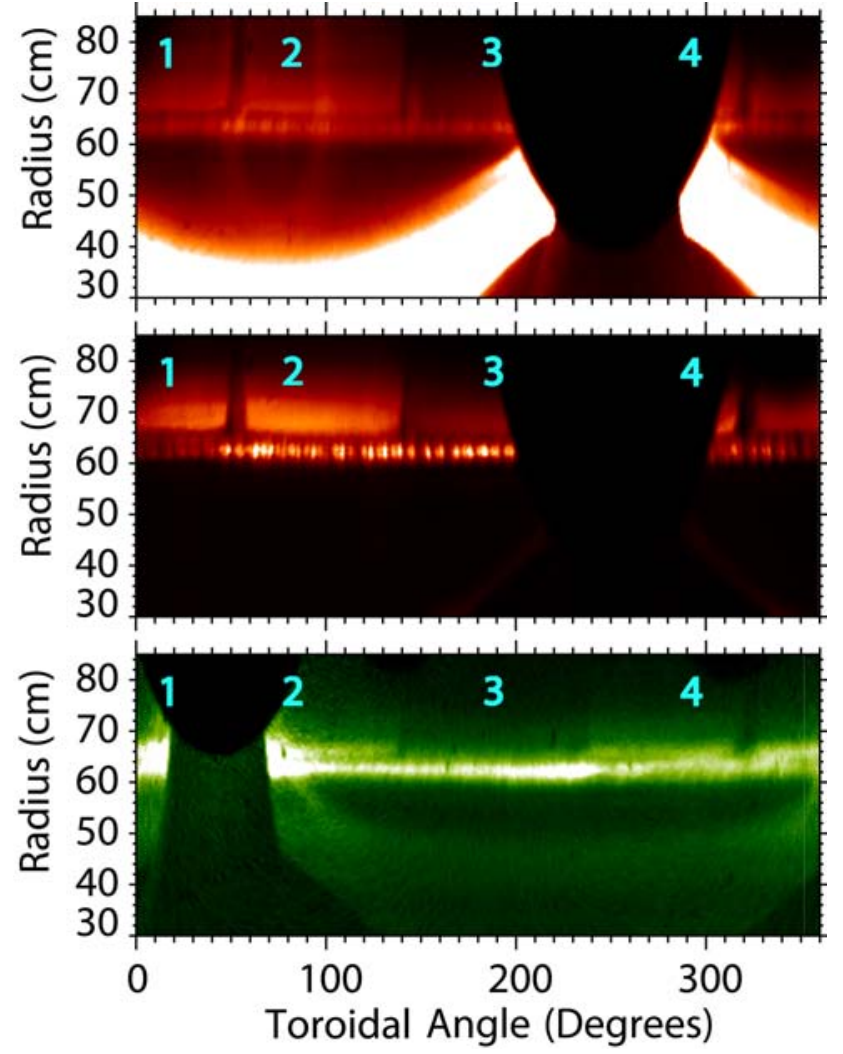

FIG. 4. a) D- $\alpha$ emission , b) Li I emission and c) Li II emission during experiments with heated LLD.

\section{B. Toroidal asymmetries in plasma surface interaction}

The full toroidal imaging allowed the study of the patterns of strike point splitting as a result of the different toroidal spectrum of perturbing $3 \mathrm{D}$ fields (e.g. $n=1$ vs $n=3$ ). For this purpose, Li I imaging was the most effective, thanks to the routine use of lithium conditioning and to the low ionization potential of lithium $(\sim 5 \mathrm{eV})$ that results in emission localized at the PFCs surface.

In NSTX, 3D magnetic perturbations produced by a set of six midplane coils are used for error field correction, resistive wall mode control and ELM triggering. The application of $3 \mathrm{D}$ fields can result in the strike point 
splitting in helical lobes of heat and particle fluxes ${ }^{3}$. In Figure 5a and 5b respectively, an unperturbed toroidally symmetric OSP and an OSP perturbed by the application of $n=3$ fields are shown. One can notice the spiral structure of particle flux lobes toroidally separated by $120^{\circ}$. Toroidally asymmetric plasma surface interactions can also occur during ELMs as shown in Figure 5c, where the Li I filter is used to image particle fluxes to the PFCs during a Type V ELM. Toroidally asymmetric auxiliary heating deposition in the plasma edge can result in the non-axisymmetric deposition of divertor heat and particles fluxes. In NSTX, radio frequency (RF) heating by High Harmonic Fast Waves (the antenna covers $90^{\circ}$ toroidally) results in a significant fraction of the injected power being coupled to the edge/SOL plasma and propagating directly to the divertor region, leading to non axisymmetric helical structures with higher particle loads (RF "hot" divertor zone) $)^{8}$. In Figure 5d, the spiral structure due to the RF divertor "hot" zone is shown as imaged by Li I filter.

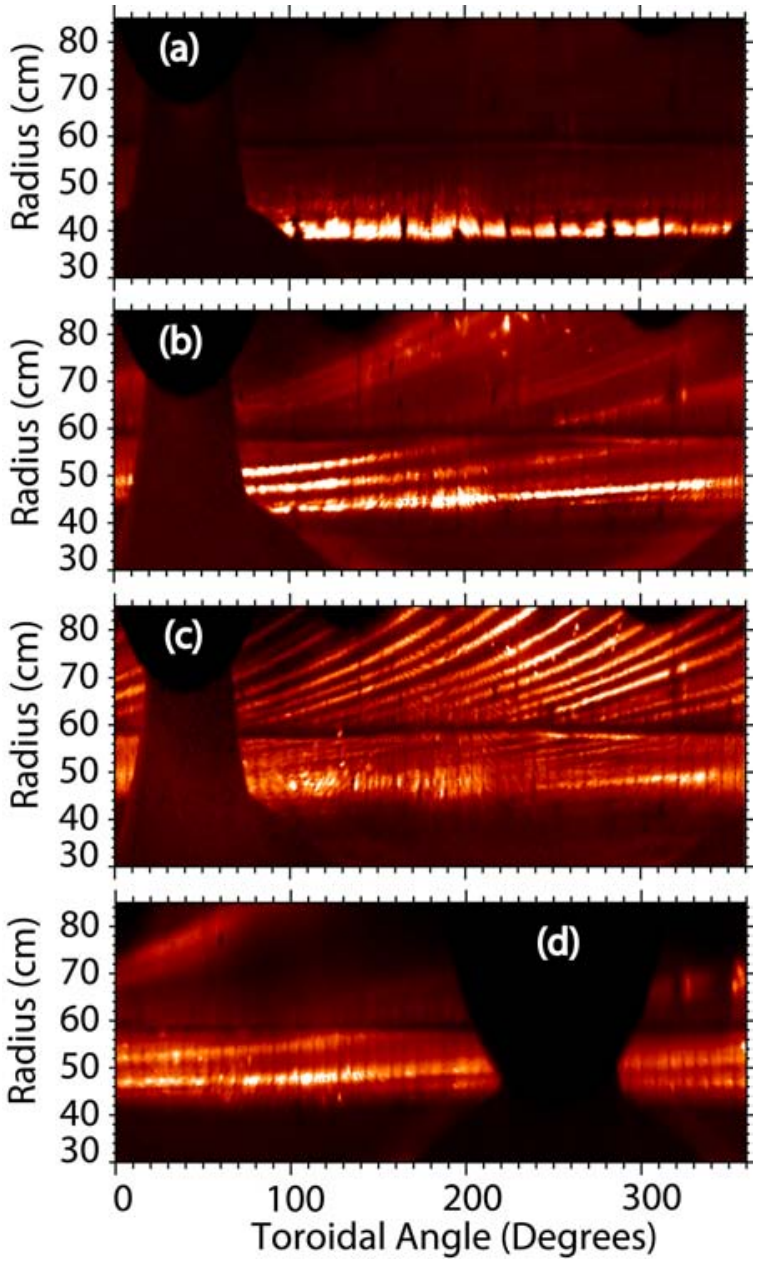

FIG. 5. Measured Li I brightness for a) unperturbed OSP, b) OSP splitting with application of $n=3$ fields, c) Type V ELM, d) RF divertor "hot" zone.

\section{Carbon sources}

Carbon represents the main core contaminant in NSTX discharges, and divertor sources are believed to be one of the main contributors. Full toroidal imaging is important to understand the carbon source distribution, as well as possible LLD contamination by sputtered carbon when the OSP is on the LLD. As an example, in Figure $6 \mathrm{a}$ and $6 \mathrm{~b}$, measured C III brightness in a discharge with OSP on inboard divertor graphite tiles and in a discharge with OSP on the LLD are shown. When the OSP is on the inboard graphite tiles (Figure 6a), C III emission is toroidally uniform. When the OSP is on the LLD (Figure $6 \mathrm{~b})$, the presence of different materials and leading edges results in toroidally asymmetric carbon influxes at $\mathrm{R}=65 \mathrm{~cm}$ and $\phi=55,145,325^{\circ}$.

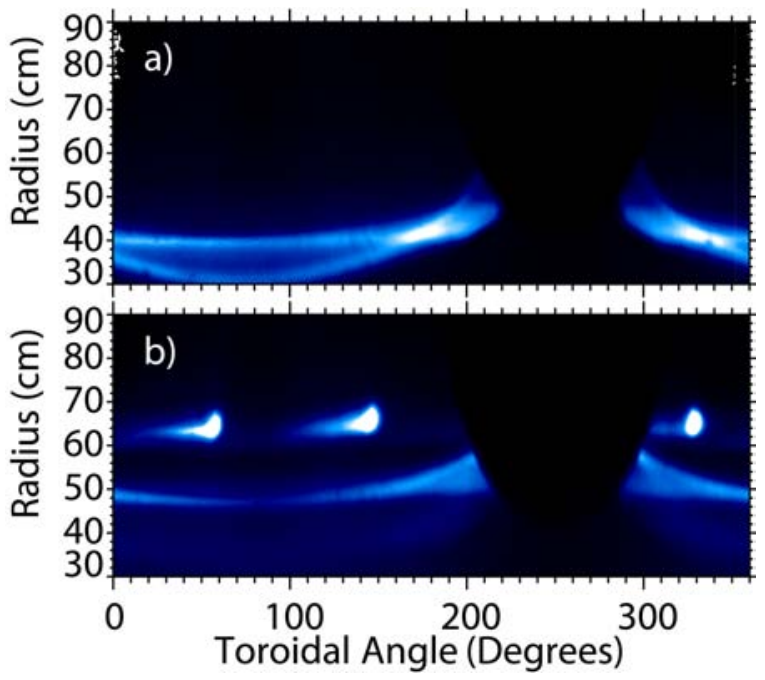

FIG. 6. C III brightness measured for a) high triangularity discharge with OSP on inboard divertor, b) low triangularity discharge with OSP on LLD.

\section{ACKNOWLEDGEMENTS}

The authors would like to acknowledge Dr. R. Maqueda for help in setup and software development, Dr. R.E. Bell for useful discussions and W. Davis for computer and technical support. This work was supported by U.S. DOE Contract DE-AC02-09CH11466 and DEAC52-07NA27344

${ }^{1}$ M.G. Bell, H.W. Kugel, et al. Plasma Phys. Controlled Fusion, 51(12), 2009.

${ }^{2}$ H.W. Kugel, M.G. Bell, et al. J. Nucl. Mater., 415 (2011), S400S404.

${ }^{3}$ J.-W. Ahn, J.M. Canik, V.A. Soukhanovskii, R. Maingi, and D.J. Battaglia. Nucl. Fusion, 50(4):045010, 2010.

${ }^{4}$ www.andover.com.

${ }^{5}$ K.H. Behringer. J. Nucl. Mater., 145-147:145-153, 1987.

${ }^{6}$ L.E. Zakharov. Private communication.

${ }^{7}$ J. P. Allain, M. D. Coventry, and D. N. Ruzic. Phys. Rev. B, 76:205434, Nov 2007

${ }^{8} \mathrm{R}$. Perkins, et al. submitted to Phys. Rev. Lett. 